Finally, the authors report that their practice is to perform a laser cone biopsy when cytological abnormality persists or worsens after a normal colposcopic biopsy. We share their concern that women with abnormal cervical cytology should be rigorously followed up after a negative punch biopsy. As our paper questioned the effectiveness of colposcopically directed punch biopsy in excluding disease, however, we believe that further information on the diagnostic yield of laser cone biopsy in these circumstances would be extremely valuable.

Paul Byrne

Pathology Laboratory,

Warwick CV34 5BJ

Department of Social Medicine,

Ciaran WoOdman

$$
\text { Medical School }
$$

Birmingham B15 2TJ

1 Singer A, Walker P, Tay SK, Dyson J. Impact of introduction of colposcopy to a district general hospital. Br Med $\mathcal{F}$ 1984;289. colposcopy

2 Meisels A, Fortin R, Roy M. Condylomatous lesions of the cervix. II. Cytologic, colposcopic and histopathologic study. Lia (ivol 1977;21:379-90)

3 Purola E, Savia E. Cytology of gynecologic condyloma acuminatum. Acta Cytol 1977;21:26-31

4 Meisels A, Morin C, Casas-Cordero M. Human papillomavirus infection of the uterine cervix. Int I Gynecol Pathol 1982;1: $75-94$

5 Jenkins D, Tay SK, McCance DJ, et al. Histological and immunohistochemical study of cervical intraepithelial neoimmunohistochemical study of cervical intraepithelial neo-
plasia (CIN) with associated HPV 6 and HPV 16 infections. f Clin Pathol 1986:39:1177-80.

\section{Strict product liability arrives}

SIR,-Ms Clare Dyer's article (27 February, p 635) on product liability raises many important points for the medical profession at large, but particularly, as she points out, for "doctors who introduce additives into intravenous infusions, which is common practice in operating theatres and intensive care units." It is vitally important that guidelines for such uses are established and accepted by both clinicians and the pharmaceutical industry at an early stage if the interests of anaesthetists and intensivists (and consequently their patients) are not to be compromised.

As supplied some drugs are unsuitable for direct administration to the patient and must be mixed with a diluent before use. Examples would be antibiotics, using a small volume of diluent, and inotropes, using a larger volume. In these cases the manufacturer usually states the diluent and dilution required, and provided these instructions are adhered to it is difficult to envisage any responsibility devolving on to the clinician. Such agents are, however, often diluted differently by individuals or units, who should confirm the suitability of their practice directly with the manufacturer. Other drugs-for example, some muscle relaxants-are supplied ready to use. It may be desirable to administer these by infusion, which can be achieved with the undiluted drug only when a suitable syringe driver is available. As this is not always the case, further dilution may be required. In these instances it would be necessary for the manufacturer to state on the datasheet suitable methods of dilution. Such practice would probably be considered a normal mode of administration rather than the constitution of a new product. The use of a simple diluent such as $0.9 \%$ sodium chloride or water for injections, and limiting such mixtures to single drugs, would minimise the likelihood of drug interactions or inactivation in vitro.

On the other hand, the Consumer Protection Act probably means that there is no longer any place for the ad hoc mixture of drugs in one container. Uncertainty about the resulting pharmacological constitution, physicochemical characteristics, and stability of such mixtures will almost certainly result in their being considered new products and the clinician a producer. While this practice is unnecessary in many instances, it is widespread among anaesthetists_-for example, in the use of atropine and neostigmine for reversing muscle relaxation. Some such mixtures are becoming commercially available, although they have the possible disadvantage that dosage ratios are fixed.

More generally, the widespread use of generic prescribing and dispensing, whether in hospital or general practice, inevitably makes it more difficult to keep a record of the source and batch number of a particular drug used in any patient. Anaesthetists are generally exacting in their records, yet these consist mostly of approved names, not trade names; indeed it is difficult to see how any of the proposed additional information could be recorded in prac tice, unless by the use of some automated system such as a barcode reader.

South Cleveland Hospital,

Middlesbrough, Cleveland TS3 4BW

Nigel Puttick

\section{Dietary management of gastroenteritis}

SIR,-We are surprised that the leading article by Dr B A Wharton and others on the dietary management of gastroenteritis (13 February, p 450) dismisses the use of a low lactose special formula without mentioning the use of a milk based low lactose formula, which has been shown to speed recovery from gastroenteritis. ${ }^{1}$ We have not seen such a dramatic reduction in lactose intolerance after gastroenteritis as the author of an editorial in the Lancet.'

Until three months ago our policy on the management of gastroenteritis in bottle fed infants was similar to that recommended by Dr Wharton and colleagues. We then changed our management to give $5 \%$ dextrose feeds for 12 hours followed by a full strength low lactose milk based formula (HN25, Milupa Ltd, Denham, Middlesex). This is continued for 10-14 days and then normal formula feeds are reintroduced.

In the three months since the new policy was introduced we have treated 37 infants with mild gastroenteritis not requiring intravenous therapy. The formula was well tolerated without vomiting in all but one infant. Diarrhoea ceased within 12 hours and only one infant relapsed when the special formula was stopped by the general practitioner and normal formula feeds reintroduced afte only four days.

During the same period one year ago 67 infants were treated for mild gastroenteritis. In 14 infants diarrhoea returned immediately feeds were reintroduced and these were given soya based lactose free formulas. A further four infants relapsed after discharge home and required readmission to hos pital. There was no difference in the average length of hospital stay of the two groups.

Our preliminary conclusion is that the use of low lactose milk based special formula is beneficia in the treatment of mild gastroenteritis and reduce the risk of relapse of diarrhoea.

\section{DAVID N K SYMON JOAN STEVENSON LESLEY PEARSON PATRICIA G P SANDERSON}

General Hospital

Hartlepool TS24 9AH

1 Howard FM, O'Halloran ET, Creagh A. Diarrhoea: after rehydration, what next? Hum Nutr A ppl Nutr 1985;39A:53-61. Anonymous. What has happened to carbohydrate intolerance following gastroenteritis? [Editorial.] Lancet 1987;i:23-4.

\section{Refusing to treat the indigent}

SIR,-Minerva's suggestion that a patient in the USA would be asked about his hospital insurance before being given tissue plasminogen activator for acute myocardial infarction is a misunderstanding of the quotation. Usually statistical data-name, address, phone number, next of kin, social security number, insurance carrier, etc-are asked for before treatment. In an emergency such as an acute myocardial infarction, however, the need for treatment pre-empts such information gathering.

The hospital cost of treatment with tissue plasminogen activator for acute myocardial infarction is $\$ 2300$, not the $\$ 10000$ to $\$ 15000$ quoted, and it is already available in an overwhelming majority of local United States hospitals.

To withhold medical care in an emergency on the grounds of inability to pay is unacceptable in American medicine. To do so would be to invite multi-million dollar malpractice suits, with the expectation of large punitive damages, and it would bring down the wrath of the local peer review organisation. The doctors concerned could be banned from treating any patient paid for by federal funds-over $40 \%$ of hospital patientsand could be reported to the state health department, which might cancel the doctor's licences to practise. Federally mandated peer review organisations examine the records of every patient who dies in hospital, along with those of all patients injured in hospital accidents, including those affected by allergic reactions to drugs and bedsores, patients readmitted within 30 days of discharge those who make an unscheduled return for surgery, etc, along with a random sample of other patients. Sanctions are applied if the care is substandard. If a pattern of refusal to care for indigent patients were discovered the matter would be referred to the state health department, which would then impose sanctions ranging from a fine to removal of the hospital's operating certificate and closure of the hospital.

Patchogue,

M G JaCOBY

\title{
Genetic counselling
}

SIR,-The two personal view articles by Professor Michael Drummond and the pseudonymous Louise Knight (20 February, p 566 and p 567) are a graphic illustration of the problems of obtaining informed consent for procedures in which the outcomes have not only low probabilities but a wide range of utilities. Your correspondents report one 38 year old woman who was not offered amniocentesis for Down's syndrome and was later worried that she might have accepted it if it had been. Another woman aged 35 was offered amniocentesis in an apparently neutral way and accepted it. She later concluded that this was the wrong decision for her since she probably values the accidental abortion of a normal child as much worse than the birth of a child with Down's syndrome. The situation was complicated in the second case because the woman became aware of a further possible risk from amniocentesis which preyed on her mind. Both women went on to deliver normal healthy babies yet were dissatisfied with the care they received. What can the obstetrician do?

Genetic counsellors do not usually know the patient's utilities before describing the possible outcomes and probabilities of a course of action. Without detailed counselling patients will often make wrong decisions because of faulty information. Counselling, however, may influence patients to make decisions not in accord with their 
true values because of a faulty heuristic. For example, a patient may accept an offered test because she reasons that the doctor would not have offered it if he did not think she should accept it. Another patient may argue that if a test is not offered then the doctors must have decided that for her it would not be advisable. If we assume that economic considerations can be disregarded the truth in all cases is that for every woman the decision depends not only on her genetic risk and the risks of the procedure but also on the relative utility she assigns to accidently losing a normal baby and to having a child with Down's syndrome. Methods for measuring people's utilities have been described but are time consuming.' Surveys of values measured in a standardised way may alert doctors to the wide range of patients' utilities but are of little use in an individual consultation.

This subject could usefully be discussed by women and their peers in early antenatal classes. The probabilities of various outcomes can be given and the women introduced to the concept of utility, whereby different patients with identical risks might choose different treatments. A reasonably sized group should contain patients with utilities at both ends of the range, or a counsellor could describe patients with utilities not represented without the danger of exerting undue influence on the suggestible. Most patients should then be able to decide whether to undergo amniocentesis without any further individual counselling. We are presently studying whether such an approach does indeed result in improved decisions.

J G THORNTON

Clarendon Wing,
Leeds General Infirmary,

Leeds LS2 9NS

R J LILFORD

St James Hospital

Leeds LS9 7TF

1 Pauker SP, Pauker SG. Prenatal diagnosis: a directive approach to genetic counselling using decision analysis. Yale $\mathcal{f}$ Biol Med 1977;50:275-89.

\section{Who needs pulse oximetry?}

SIR,-Following Dr John S M Zorab's leading article (5 March, p 658) we would like to describe a recent case in which we used oximetry for measuring oxygen saturation during the transport of a sick patient.

After the fire in a hotel in Borovets, Bulgaria, in February all the injured from the United Kingdom were flown home two days later. They included one patient who had had a laparotomy, splenectomy, and a tear in the liver sutured and packed. The patient had also sustained a comminuted fracture of a femur, which was in traction. Resuscitation had included 5 units of blood. The most recent haemoglobin value was $116 \mathrm{~g} / \mathrm{l}$. There were symptoms of chestiness, probably as a result of smoke inhalation. There was no sign of fat embolism. These injuries, and those of the rest of the casualties, had been sustained in jumping from the third and fourth floors of the hotel.

A pulse oximeter (Novametrix) was attached to a finger in the ambulance outside the hospital in Samokov, $70 \mathrm{~km}$ from Sofia, and removed at Gatwick. It was used frequently but not continuously. The altitude at Samokov is around 3000 feet $(910 \mathrm{~m})$ and at Sofia (the airport) 2000 feet $(610$ $\mathrm{m}$ ), and the cabin altitude during flight was 5000 feet $(1520 \mathrm{~m})$. Oxygen saturation while the patient was breathing air rose during the drive down from the hills, from $85 \%$ to $89 \%$, proving that supplementary oxygen would be needed during the flight. This was given, the flow being titrated to give an oxygen saturation of $95-97 \%$. When the mask was removed the saturation fell promptly to $85 \%$ or less.

This instrument was of great value during this medical repatriation, helping to provide a safer environment for the patient and reassurance for the escorts.

ROBIN M WELLER

Anaesthetic Department,

Frenchay Hospital,

Bristol BS16 ILE

SIR,-Dr John Zorab's leading article on pulse oximetry (5 March, p 658), by quoting Severinghaus and Naifeh, ${ }^{1}$ may leave the reader with the impression that pulse oximeters vary widely in the accuracy of calculated arterial blood oxygen saturation. This paper, however, was evaluating the response of pulse oximeters to a brief period of induced profound hypoxia (arterial oxygen saturation $40-70 \%$ ).

While there are significant limitations to the accuracy of pulse oximeters, ${ }^{2}$ and this remains an area of concern and for future development, ${ }^{3}$ our recent study directly comparing the steady state accuracy of pulse oximeters in the clinically useful range (arterial oxygen saturation $80-100 \%$ ) was more encouraging. ${ }^{4}$ With one exception all the oximeters showed little variation in calculated arterial oxygen saturation, but had a general tendency slightly to underestimate the true value. Accuracy in this range is to be expected as manufacturers calibrate pulse oximeters empirically, deriving an algorithm from in vivo studies.

In many, but not all, of the clinical situations in which pulse oximeters may be used a consistent accuracy of $2-3 \%$ SD in the $80-100 \%$ arterial oxygen saturation range is probably acceptable as long as falling or rising trends are reliably detected. There is some evidence that ear probes may detec such trends more rapidly and accurately than finger probes.

M B TAYLOR

Department of Anaesthetics,

J G WHITWAM

Royal Postgraduate Medical School,

London W $120 \mathrm{HS}$

Severinghaus JW, Naifeh KH. Accuracy of response of six pulse oximeters to profound hypoxia. Anesthesiology 1987;67:551-8.

Taylor MB, Whitwam JG. The current status of pulse oximetry.

Clinical value of non-invasive oxygen saturation monitoring. Clinical value of non-invasive

3 Huch A, Huch R, Konich V, et al. Limitations of pulse oximetry. Lancet 1988;i:357-8.

4 Taylor MB, Whitwam JG. The accuracy of pulse oximeters. A comparative clinical evaluation of five pulse oximeters. Anaesthesia 1988;43:229-32.

5 Kagle DM, Alexander CM, Berko RS, Giuffre M, Gross JB. Evaluation of the Ohmeda 3700 pulse oximeter: steady-state and transient response characteristics. Anesthesiology 1987;66: 376-80.

\section{Does infection with HIV affect the} outcome of pregnancy?

SIR,-Dr Frank Johnstone and others examine the outcome of pregnancy in women who either were intravenous drug users or had a seropositive partner (13 February, p 296). The outcome of pregnancy in the HIV seropositive and seronegative groups were compared. Apart from an increased rate of spontaneous abortion in the seropositive group there were no appreciable differences between the two groups; in both there were increased rates of adverse outcome in terms of preterm deliveries and small for gestational age and low birthweight babies. Since the data are considered from the viewpoint of HIV infection it is not possible to assess the influence on pregnancy outcome of intravenous drug abuse, but it can be inferred that this is one of the features of "lifestyle and deprivation" to which the adverse outcomes were attributed.

Although HIV infection is not yet a problem in obstetric practice in Glasgow, intravenous drug abuse is common. Since February 1986 pregnant intravenous drug abusers and partners of drug users in the north of Glasgow have been cared for at a community based antenatal clinic which operates in close liaison with community health services, the social work department, and the local drugs project, as well as other hospital specialties. Since HIV infection is a potential risk of intravenous drug use, which in turn correlates strongly with socioeconomic deprivation, this system provides a comprehensive service dealing with all aspects of deprivation. Initial management of drug abuse is by immediate or very rapid detoxification as an inpatient despite the fact that this is reportedly associated with increased fetal loss.

By 31 January 1988, 28 women with a history of intravenous drug use and 17 partners of men with this history had been delivered. All were HIV seronegative. Of the 28 , only two had stopped using drugs before pregnancy; at the time of referral many were using large quantities of drugs with many consequent medical problems. There were no spontaneous abortions and one induced abortion for fetal abnormality; this woman was one of the two who had stopped using drugs before pregnancy. Three babies were delivered at $<37$ weeks' gestation, three had birthweights $<2500 \mathrm{~g}$, and one had a birthweight $<5$ th centile. Among the 17 partners of drug users there was one spontaneous abortion, one baby delivered at $<37$ weeks' gestation, two with birthweights $<2500 \mathrm{~g}$, and one with a birthweight $<5$ th centile. There were no perinatal deaths in either group.

The comprehensive management described here has many benefits, not least being its acceptability to the women it serves. Management of drug abuse in pregnancy by immediate detoxification allows an earlier start to rehabilitation, avoids withdrawal symptoms in the babies, and thus reduces the risk of separation of mother and baby at birth. Although the numbers are small, our results suggest this form of management may not be as hazardous to the fetus as earlier reports would suggest.

MARY HEPBURN
COLIN A Forrest

Division of Obstetrics and Gynaecology,

Stobhill General Hospital,

Glasgow G21 3UW

\section{Antisperm antibodies in infertility}

SIR,-In Mr David Barlow's leading article the role of direct intraperitoneal insemination ${ }^{1}$ in inducing the production of antisperm antibodies was questioned (30 January, p 310).

Direct intraperitoneal insemination has been performed at St Mary's Hospital, Manchester, since January 1987. Ovulation is stimulated by clomiphene citrate at $100 \mathrm{mg} /$ day on days 2-6 of the menstrual cycle and triggered by an injection of human chorionic gonadotrophin (10000 IU intramuscularly) when the leading follicle is $20 \mathrm{~mm}$ in diameter or by the occurrence of an endogenous luteinising hormone surge detected in serum samples collected daily. Washed motile sperm (mean $12.1 \times 10^{6}$; range $3.2-22.0 \times 10^{6}$ ) in $0.5 \mathrm{ml}$ culture medium is injected into the peritoneal cavity through the vaginal wall $34-36$ hours after the ovulatory trigger using a $1 \mathrm{ml}$ syringe and 21 gauge needle.

Blood is collected from the female partner in the follicular phase of the treatment cycle (day 2-5), and 14 days and 28 days after the direct intraperitoneal insemination, and serum is tested for 